\title{
Simulation of single transparent molecule interaction with an optical microcavity
}

\author{
Haiyong Quan and Zhixiong Guo ${ }^{1}$ \\ Department of Mechanical and Aerospace Engineering, Rutgers, the State University of New \\ Jersey, Piscataway, NJ 08854, USA \\ E-mail: guo@jove.rutgers.edu
}

Received 9 May 2007, in final form 24 July 2007

Published 22 August 2007

Online at stacks.iop.org/Nano/18/375702

\begin{abstract}
Finite-element simulations of nanoscale molecule interaction with the evanescent radiation field of an optical resonant microcavity are conducted to characterize the detection of single transparent molecules using the microcavity as an extremely sensitive micro/nano-sensor. The model sensor is an integrated device consisting of a dielectric microdisk and a waveguide that can be nanofabricated on Si-based dielectric thin film. When the microdisk is operated at a whispering-gallery mode, a strong evanescent field arises, surrounding the periphery of the cavity. Foreign target molecules such as proteins present in the near-field will interact with the electromagnetic resonant field and induce changes to the resonance. Such induced changes are investigated in this report and their significance in the detection of single molecules for nanotechnology development is discussed.
\end{abstract}

(Some figures in this article are in colour only in the electronic version)

\section{Introduction}

Whispering-gallery mode (WGM) occurs when light travels in a dielectric medium of circular geometry. After repeated total internal reflections at the curvilinear boundary the electromagnetic field can close on itself, giving rise to resonances. WGM resonances possess very high quality factors due to minimal reflection loss. If the resonating cavity is in the microscale, one obtains a very small mode volume and high finesse. Devices featuring resonant microcavity structures have high potential for the realization of cavity quantum electrodynamics [1], microlasers [2], selective and tunable filters [3], optical switching [4], miniature biosensors [5], and high resolution spectroscopy [6]. The increasing demands for compact devices to detect biomolecules open up new research opportunities and challenges.

Near-field radiation plays an important role in micro/nanoscale diagnostics [7, 8]. Evanescent radiationmolecule interactions provide novel concepts and innovative tools for the exploration of the molecular world and the development of nanotechnology. Optical techniques for single molecule detection have recently attracted much attention in

\footnotetext{
1 Author to whom any correspondence should be addressed.
}

the field of life sciences. Optical methods in the far-field cannot localize the dimension to better than the diffraction limit. To overcome this restriction, near-field optical techniques are widely and successfully utilized to study the dynamic properties of biological molecules. One highly successful example is near-field scanning optical microscopy (NSOM). Recent advances in NSOM in combination with fluorescence techniques have allowed for imaging [9], dynamics [10], spectroscopy [11], and resonance-energy transfer [12] of single molecules. Direct detection of nanoscale transparent entities without labeling is still a challenge, but may be resolved through the use of extremely sensitive WGM signals [13].

In this study, we will simulate the interaction of a single transparent biomolecule with the evanescent radiation field of a dielectric microcavity. The interaction is governed by Maxwell's electromagnetic (EM) theory. The finite-element method is employed for solving the Helmholtz equations with appropriate boundary conditions. The simulation model is verified through benchmark comparisons with analytical and other published numerical results. The induced resonant condition changes, such as frequency shift and intensity variation, due to the molecule-radiation interaction are investigated. 


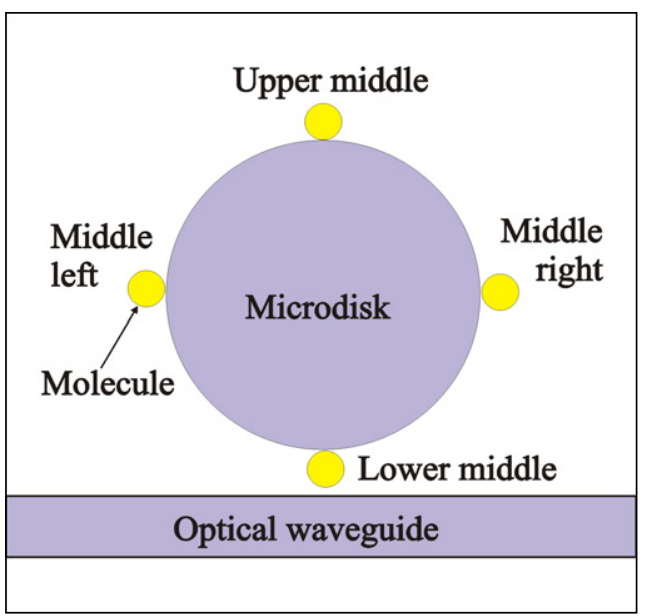

Figure 1. Sketch of a WGM microcavity simulation domain and locations of single molecules.

\section{Modeling}

Consider a dielectric microdisk coupled with a light-delivery waveguide as shown in figure 1. A small air-gap separates the microcavity from the waveguide. The microdisk and waveguide are made of the same material (silicon nitride) and are assumed to have a constant refractive index of 2.01 against the excitation wavelengths [14]. For the sake of calculations, the foreign target molecules that are present in the evanescent field are assumed to be of circular geometry. A molecule can approach the microcavity from any direction. Here we only consider four exemplary directions, i.e., a molecule is located in either the upper middle, lower middle, middle left or middle right position to the microcavity.

The molecule-radiation interaction is governed by Maxwell's equations. By introducing time-harmonic waves, Maxwell's equations can be reduced to Helmholtz equations as follows:

$$
\begin{aligned}
\frac{1}{\mu} \nabla^{2} \bar{E}+\omega^{2} \varepsilon_{\mathrm{c}} \bar{E} & =0 \\
\frac{1}{\mu} \nabla^{2} \bar{H}+\omega^{2} \varepsilon_{\mathrm{c}} \bar{H} & =0
\end{aligned}
$$

where $\bar{E}$ and $\bar{H}$ are the electric and magnetic field vectors, respectively; and $\omega=2 \pi c / \lambda . \quad c$ is the speed of light in the medium and $\lambda$ is the light wavelength. The complex permittivity is $\varepsilon_{\mathrm{c}}=\varepsilon_{\mathrm{cr}} \varepsilon_{0}=\varepsilon-\mathrm{i}(\sigma / \omega)$, where $\varepsilon_{\mathrm{cr}}$ is the complex relative permittivity, $\varepsilon_{0}$ is the permittivity in vacuum and $\sigma$ is the electrical conductivity. If the complex index of refraction, $m=n-\mathrm{i} k$, is given, the complex relative permittivity can be obtained through the relationship: $\varepsilon_{\mathrm{cr}}=$ $m^{2}=n^{2}-k^{2}-\mathrm{i} 2 n k$. The absorption index $k$ for dielectric media is extremely small and negligible.

In the present study we consider the in-plane TE waves, where the electric field has only a $z$-component; and it propagates in the $x-y$ plane (disk-waveguide plane). Thus, the fields can be written as:

$$
\begin{gathered}
\bar{E}(x, y, t)=E_{z}(x, y) \bar{e}_{z} \mathrm{e}^{\mathrm{i} \omega t} \\
\bar{H}(x, y, t)=\left[H_{x}(x, y) \bar{e}_{x}+H_{y}(x, y) \bar{e}_{y}\right] \mathrm{e}^{\mathrm{i} \omega t} .
\end{gathered}
$$

Table 1. Comparison of the theoretical and numerical resonance wavelengths for an isolated $2 \mu \mathrm{m}$-diameter cavity.

\begin{tabular}{llll}
\hline $\begin{array}{l}\text { Azimuthal } \\
\text { mode } \\
\text { number } n\end{array}$ & $\begin{array}{l}\text { Theoretical } \\
\text { resonance } \\
\text { wavelength (nm) }\end{array}$ & $\begin{array}{l}\text { Numerical } \\
\text { resonance } \\
\text { wavelength (nm) }\end{array}$ & $\begin{array}{l}\text { Relative } \\
\text { error }(\%)\end{array}$ \\
\hline 8 & 1170.548 & 1168.92 & 0.141 \\
10 & 964.891 & 964.110 & 0.081 \\
12 & 822.9948 & 822.964 & 0.004 \\
14 & 718.755 & 718.709 & 0.006 \\
16 & 638.6908 & 638.638 & 0.008 \\
18 & 575.1338 & 575.067 & 0.012 \\
20 & 523.3831 & 523.2915 & 0.018 \\
22 & 480.2857 & 480.2722 & 0.003 \\
\hline
\end{tabular}

Table 2. Comparison of resonance frequencies between the predictions of FEM and FDTD for the $5 \mu$ m-diameter cavity coupled with two waveguides (FDTD data are cited from [15])

\begin{tabular}{llll}
\hline \multirow{2}{*}{$\begin{array}{l}\text { Radial mode } \\
\text { number }\end{array}$} & \multicolumn{2}{l}{ Resonance frequency $(\mathrm{THz})$} & \\
\cline { 2 - 3 } & FDTD & FEM & Relative error $(\%)$ \\
\hline 1 & 189.2 & 189.42 & 0.12 \\
2 & 191.3 & 191.52 & 0.12 \\
3 & 187.8 & 188.04 & 0.13 \\
\hline
\end{tabular}

At the interface and physical boundaries, the natural continuity condition is used. For the outside boundaries, the low-reflecting boundary condition is adopted. Low-reflecting means that only a small part of the wave is reflected, and that the wave propagates through the boundary almost as if it were not present. This condition can be formulized as

$$
\bar{e}_{z} \cdot \bar{n} \times \sqrt{\mu} \bar{H}+\sqrt{\varepsilon} E_{z}=0 .
$$

The light source term $E_{0 z}$, which propagates inwards through the entry of the waveguide, is treated as an electrically low-reflecting boundary and is expressed by

$$
\bar{e}_{z} \cdot \bar{n} \times \sqrt{\mu} \bar{H}+\sqrt{\varepsilon} E_{z}=2 \sqrt{\varepsilon} E_{0 z} .
$$

Versatile numerical approaches can be employed for solving the EM problem. The finite-difference time-domain (FDTD) method [15] and the finite-element method (FEM) are among the most popular. The FEM [16] is very flexible in terms of the treatment of irregular configurations such as the circular geometry of the microcavity. Thus, the in-plane TE waves application mode of the commercial FEMLAB package (version 3.0) is employed for the finite-element analysis in the current simulations. The description of the numerical method is available in the literature $[13,16]$, and thus, is not repeated here.

The present FEM simulation is validated through close benchmark comparisons with analytical and/or published FDTD simulation results in tables 1 and 2. The resonance wavelengths of FEM simulation in table 1 for a $2 \mu \mathrm{m}$ diameter cavity made of silicon nitride are obtained under the asymptotic limit, i.e., the cavity is positioned far away from its coupling waveguide. The analytical results are from Quan [17] who used the method of separation of variables with appropriately defined boundary conditions to find the intrinsic resonance wavelengths of the given cavity without a waveguide. The FEM model is also used to simulate the 
resonance of a $5 \mu \mathrm{m}$-diameter cavity demonstrated by Taflove and Hagness [15] who used the FDTD simulation method. In both simulations, the calculation domain is a $6 \mu \mathrm{m} \times 7 \mu \mathrm{m}$ rectangular area. The microcavity is coupled to two straight waveguides at the top and bottom, respectively. The width of both the waveguides is $300 \mathrm{~nm}$ and the gap between the microdisk and either waveguide is $232 \mathrm{~nm}$. The material of the microdisk and waveguides is GaAlAs, which has a refractive index of 3.2 for the considered wavelength range. The resonance frequencies for radial modes 1,2 , and 3 with the same azimuthal mode number of 27 are listed in table 2 for comparison. Excellent agreement was found between the analytical and FEM methods and the FEM and FDTD methods. We also compared their respective E-field distributions and found similar patterns among all of the methods.

\section{Results and discussion}

Under the WGM first-order resonance, an exceedingly brilliant ring forms along the boundary of a microcavity [16]. Even though the incident light is very weak, the circulation overlapping inside the mode ring obviously enhances the mode intensity and provides a strong evanescent field surrounding the resonator periphery surface. If single molecules are attached on the periphery or within the evanescent field, it is expected that they will interact with the evanescent field. As a result, the resonance conditions may alter. Understanding such induced changes is paramount to the design of innovative WGM-based micro/nano-sensors.

In the following we simulate the interaction of a single transparent molecule with a microcavity device. The device is a $2 \mu \mathrm{m}$-diameter microdisk coupled with a straight waveguide $0.5 \mu \mathrm{m}$ wide and is made of silicon nitride. The air-gap separating the microcavity and waveguide is $280 \mathrm{~nm}$ which is an optimum gap condition [14]. The base resonance wavelength of the integrated device is found to be $822.93 \mathrm{~nm}$ for first-order resonance at azimuthal mode 12, in the case without any molecule attachment. The resonance wavelength shift (RWS) due to molecule attachment is in reference to this base resonance wavelength of the device. In general, biomolecules have different shapes and sizes as well as different optical properties. The refractive index of biological components in humans varies in the range of 1.35-1.60 [18]. In the present two-dimensional simulations, the biomolecule phantoms are simplified as circles and the molecular size discretely varies from 25 to $550 \mathrm{~nm}$ in diameter. The refractive index of the target molecules considered is assumed to be 1.45 , which is a typical value for proteins [19].

Now let us investigate a single molecule of $200 \mathrm{~nm}$ in diameter which is approaching the periphery of the microcavity from four different orientations as shown in figure 1. The presence of this molecule changes the resonance wavelength and the change (i.e., RWS) should be a function of the approaching distance. The term 'molecule attachment distance' (MAD) is used here to indicate the shortest distance from a molecule to the microdisk periphery. Figure 2 shows the simulation data and exponentially fitted curve of the resonance wavelength shift versus the molecule attachment distance. It is seen that the distance from the molecule to the microcavity obviously affects the magnitude of the RWS. When the

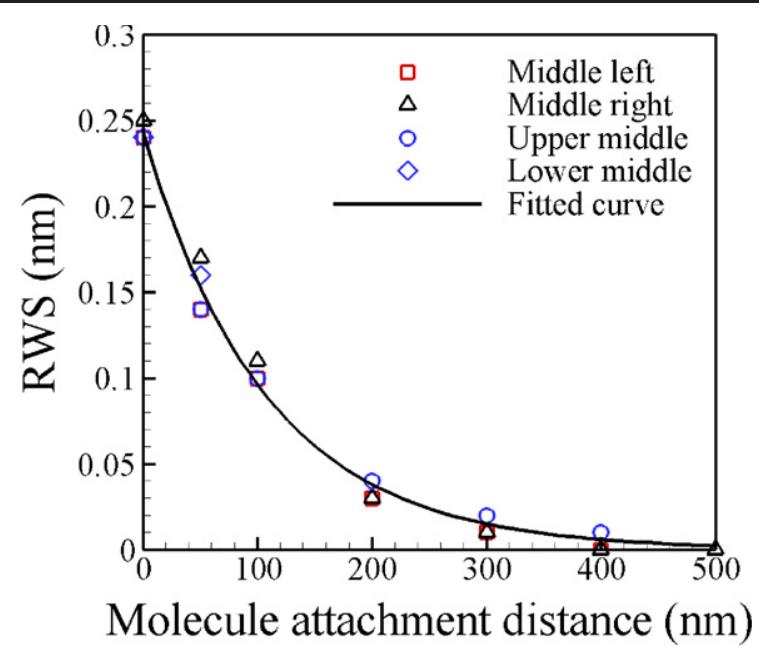

Figure 2. The induced resonance wavelength shifts (RWS) when a single molecule of $200 \mathrm{~nm}$ in diameter is approaching the $2 \mu \mathrm{m}$-diameter microcavity.

molecule is closely attached to the microcavity surface (i.e., MAD $=0 \mathrm{~nm}$ ), the induced RWS is $0.24 \mathrm{~nm}$, corresponding to a $112 \mathrm{GHz}$ frequency shift. Such a big frequency shift is certainly detectable through general optical techniques. The fitted curve reveals that the RWS decays with increasing MAD in an exponential manner. At MAD $=500 \mathrm{~nm}$, the RWS is less than $0.005 \mathrm{~nm}$. The signal change as a function of distance should be of interest for studies of biomolecular interactions and protein folding. The slight fluctuation of the simulated data for different molecule locations shown in figure 2 indicates that the effect of the molecule approaching direction is not very appreciable. This is because the EM field of a whisperinggallery mode is almost axisymmetric.

The MAD also affects the radiation intensity of the resonance mode inside the microdisk. A molecule which is closer to the microdisk may scatter more energy from the evanescent field and thus reduce the resonance intensity. Figure 3 shows the E-fields of the $2 \mu$ m-diameter microcavity sensor at resonance azimuthal mode 12 for four different molecule attachment distances. The molecule of $400 \mathrm{~nm}$ in diameter is approaching the microcavity from the upper middle position. It is seen that the resonance intensity inside the cavity weakens substantially with decreasing MAD. This indicates that the presence of a foreign molecule will reduce the quality $(Q)$ factor of resonance. In addition to the frequency shift, the $Q$ factor and the scattering radiation intensity may be exploited as signals for sensing purposes.

The molecule size effect is further disclosed in figure 4, where the stored energy inside the cavity against the diameter of the molecule at various attachment distances is plotted. With increasing molecule size, the stored energy decreases. This is consistent with the findings in figure 3 . The stored energy decreases rapidly when the molecular size increases from 25 to $150 \mathrm{~nm}$. The attachment distance is also a critical parameter to determine the intensity decay. For instance, the presence of a molecule of $500 \mathrm{~nm}$ in diameter at a MAD of $200 \mathrm{~nm}$ results in a $50 \%$ reduction of the stored energy, whereas the same molecule at zero MAD reduces the stored energy by $90 \%$. The closer the attachment, the more significant the molecule size. 


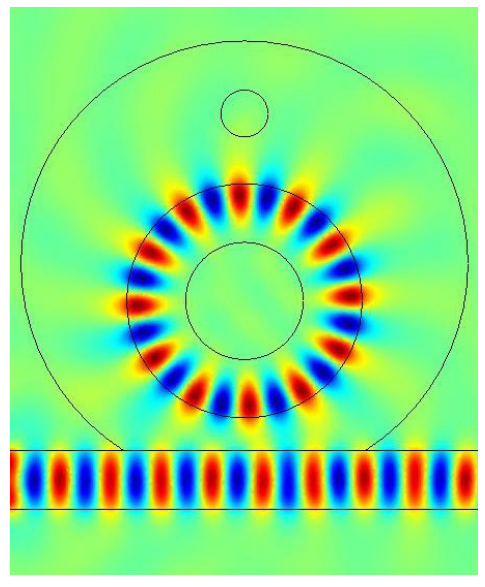

$\mathrm{MAD}=400 \mathrm{~nm}$

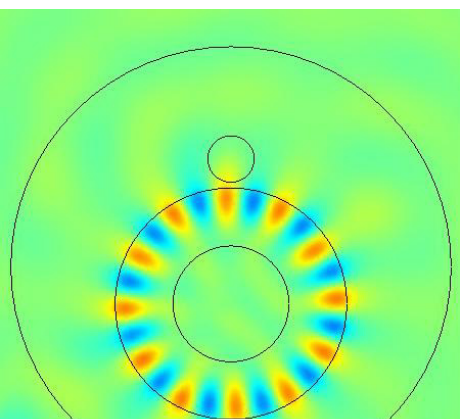

IIIIIIIIIIIIIII

$\mathrm{MAD}=50 \mathrm{~nm}$

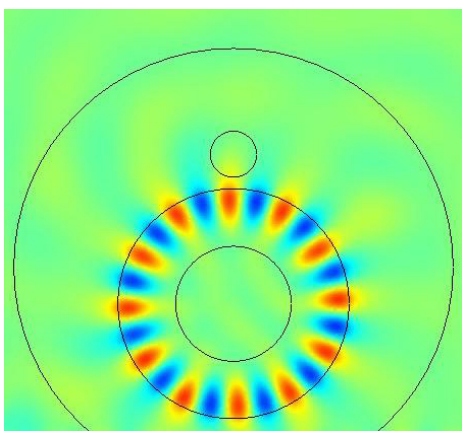

\section{IIIIIIIIIIIIIII}

$\mathrm{MAD}=100 \mathrm{~nm}$

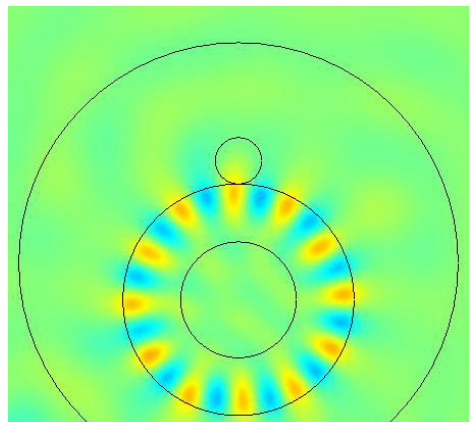

IIIIIIIIIIIIIIII

$$
\mathrm{MAD}=0 \mathrm{~nm}
$$

Figure 3. The E-field changes due to a single molecule approaching the $2 \mu$ m-diameter microcavity.

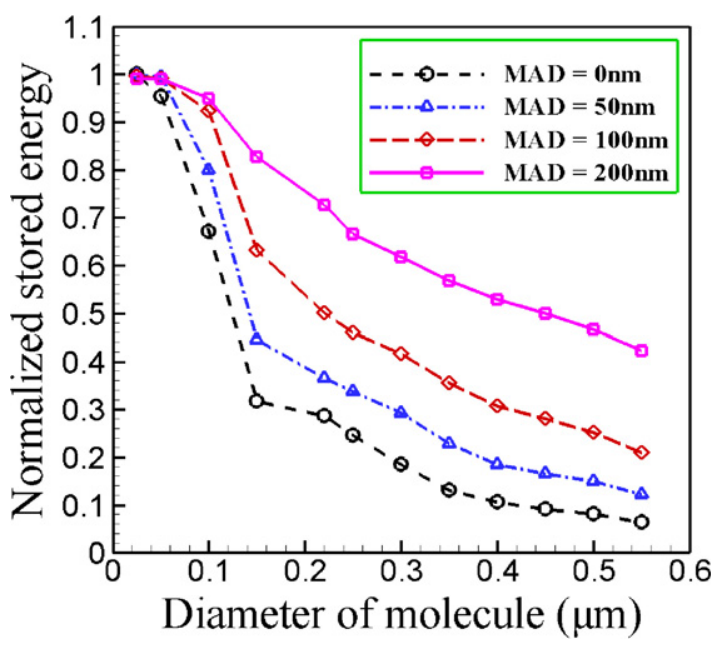

Figure 4. Molecular size effect on the stored energy inside the $2 \mu$ m-diameter microcavity.

Human tissues have very weak absorption in the nearinfrared (700-1100 $\mathrm{nm}$ ). The reported tissue absorption coefficient [20] is generally of the order of $0.01-0.1 \mathrm{~mm}^{-1}$ which corresponds to an absorption index of less than $10^{-5}$ in the complex refractive index. Such small absorption common in dielectric media is negligible when the medium is very thin, particularly at the molecular level. Thus, most biological molecules except those containing pigment are nearly transparent in the near-infrared. When absorption is significant, the resonance intensity and quality factor will be substantially lightened. Other means such as measuring transmission reduction [21] or using cavity ringdown techniques [22] could be exploited.

The present study is not only important for molecular detection, but is also of potential application in a wide range of DNA- and protein-based molecular nanotechnologies, such as the study of molecular interaction events during many biological and/or drug-delivery processes. It should be mentioned that optical microcavity-based detection techniques are not solely limited to molecules. A referee of this article brought the detection of a single atom trapped and cooled in magnetic microtraps under WGM resonance [23] to the authors' attention.

\section{Conclusion}

In summary, molecule-radiation interactions at whisperinggallery modes were simulated using the finite-element solution 
of Maxwell's equations. The FEM model was verified. Excellent agreement was found between the FEM simulation and analytical results and between the FEM and published FDTD simulations. The influence of the presence of target molecules in the near-field of the microcavity on the whispering-gallery mode resonance is investigated. It is found that a single biomolecule can affect the resonance field intensity and alter the resonance frequency/wavelength. The direction of the approaching molecule is not important. Both the molecular size and attachment distance determine the magnitude of the induced changes. With decreasing attachment distance or increasing molecule size, the shift of resonance frequency increases and the resonance field intensity weakens. By measuring these induced WGM signal changes, it is possible to detect single transparent molecules and/or to follow up molecular events. This study is significant for designing extremely sensitive biosensors for nanotechnology development.

\section{Acknowledgments}

This material is based upon work supported by the National Science Foundation under Grant No. CBET-0541585 and CBET-0651737.

\section{References}

[1] Vahala K J 2003 Nature 424839

[2] Cai M, Painter O, Vahala K J and Sercel P C 2000 Opt. Lett. 251430
[3] Boriskina S V, Benson T M, Sewell P and Nosich A I 2003 J. Lightwave Technol. 211987

[4] Blom F C, van Dijk D R, Hoekstra H J, Driessen A and Popma T J A 1997 Appl. Phys. Lett. 71747

[5] Arnold S, Khoshsima M, Teraoka I and Vollmer F 2003 Opt. Lett. 28272

[6] Schiller S and Byer R L 1991 Opt. Lett. 161138

[7] Chen G 1996 Annu. Rev. Heat Transfer 71

[8] Majumdar A 1999 Annu. Rev. Mater. Sci. 29505

[9] Meixner A J, Zeisel D, Bopp M A and Tarrach G 1995 Opt. Eng. 342324

[10] Xie X S and Dunn R C 1994 Science 265361

[11] Siegberg D, Roth C M and Herten D P 2006 Proc. SPIE $\mathbf{6 0 9 2} 609202$

[12] Ha T, Enderle T, Ogletree D F, Chemla D S, Selvin P R and Weiss S 1996 Proc. Natl Acad. Sci. 936264

[13] Quan H and Guo Z 2005 J. Quant. Spectrosc. Radiat. Transfer 93231

[14] Guo Z, Quan H and Pau S 2006 J. Phys. D: Appl. Phys. 395133

[15] Taflove A and Hagness S C 2000 Computational Electrodynamics: The Finite-Difference Time-Domain Method 2nd edn (Norwood, MA: Artech House)

[16] Guo Z, Quan H and Pau S 2006 Appl. Opt. 45611

[17] Quan H 2006 Characterization of optical whispering gallery mode resonance and applications PhD Dissertation Rutgers University (Ch. 2)

[18] Hohnsen S and Widder E A 1999 J. Theor. Biol. 199181

[19] Voros J 2004 Biophys. J. 87553

[20] Cheong W F, Prahl S A and Welch A J 1990 IEEE J. Quantum Electron. 262166

[21] Boyd R W and Heebner J E 2001 Appl. Opt. 405742

[22] Rosenberger A T and Rezac J P 2001 Proc. SPIE 4265102

[23] Rosenblit M, Horak P, Helsby S and Folman R 2004 Phys. Rev. A 70053808 